\title{
Status of the DUNE Near Detector
}

Richie Diurba (University of Minnesota) for the DUNE Collaboration

NuFACT 2021

This document was prepared by DUNE Collaboration using the resources of the Fermi National Accelerator Laboratory (Fermilab), a U.S. Department of Energy, Office of Science, HEP User Facility. Fermilab is managed by Fermi Research Alliance, LLC (FRA), acting under Contract No. DE-AC02-07CH11359.

Sanford Underground

Research Facility

800 miles
80 kilometers
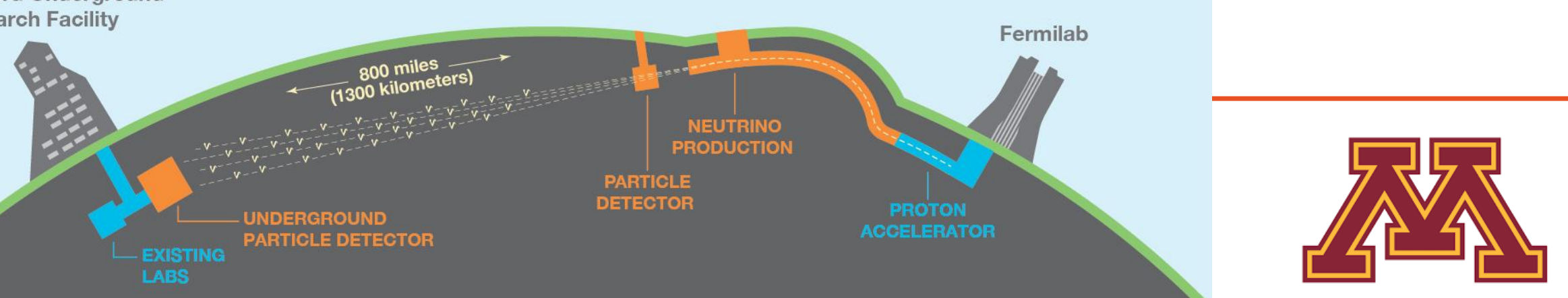


\section{Introduction to DUNE and DUNE Near Detector}

- Deep Underground Neutrino Experiment (DUNE) is a long-baseline experiment.

- Also contains atmospheric neutrino, solar neutrino, supernova, and BSM science programs.

- DUNE Near Detector allows for simultaneous understanding of the flux and neutrino interactions on argon.

- DUNE Near Detector Conceptual Design Report published in March of 2021 (arxiv:2103.13910)

Related DUNE Talks at NuFACT 2021:

- Chris Marshall, DUNE long-baseline oscillation physics sensitivity

- Matteo Vincenzi, SAND - System for on-Axis Neutrino Detection - in the DUNE Near Detector Complex

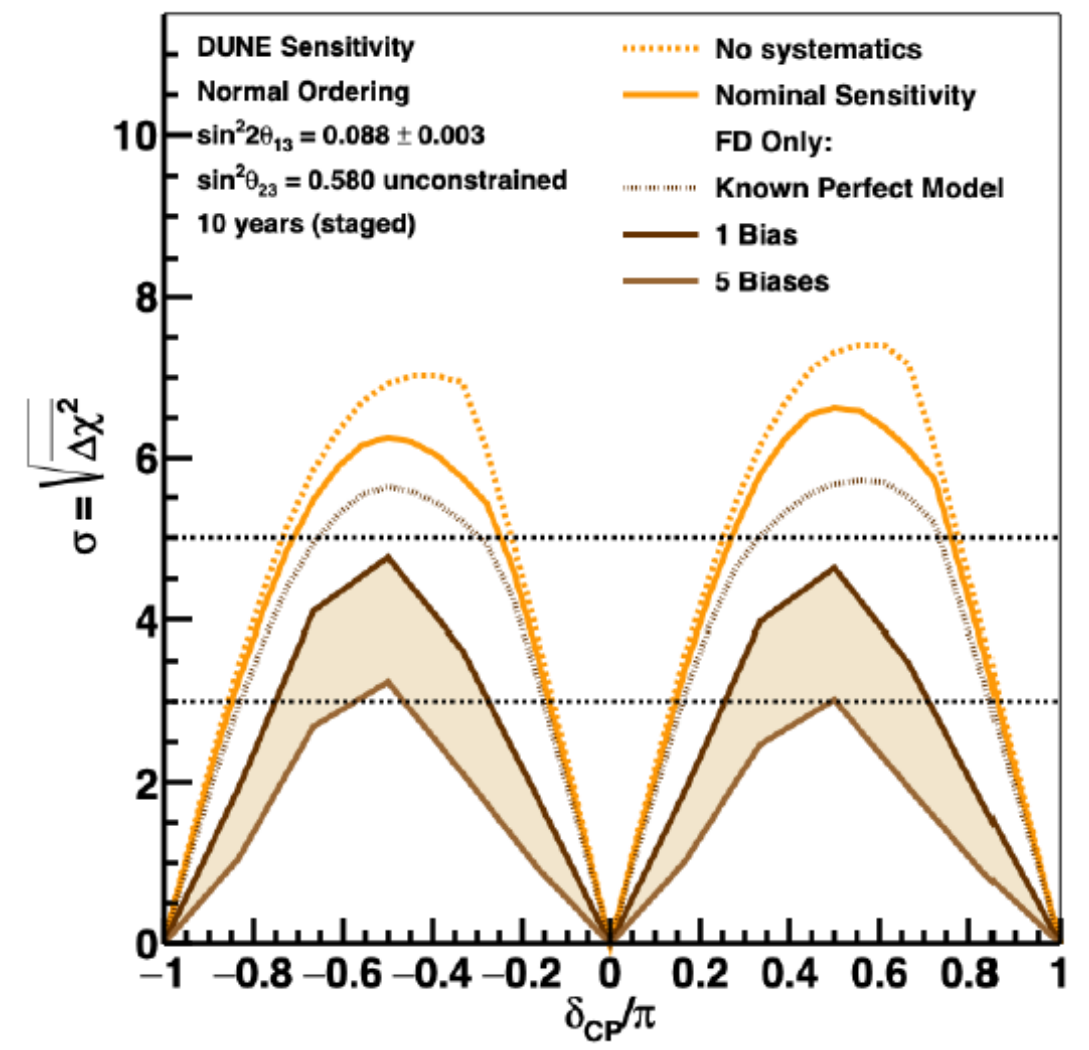

Sensitivity to detecting CP-violating phase with Far Detector only. Biases were evaluated by using GENIE and then reweighting the final data set using NuWro. "One bias" is the difference between the event generators and "five biases" is if five "one bias" differences are added in quadrature.

arxiv:2103.13910 


\section{DUNE Near Detector Complex}

Constitutes three detector systems each with important roles.

- Near Detector-Liquid Argon (ND-LAr) is a 67-ton liquid argon time projection chamber (TPC).

- Near Detector-Gaseous Argon (ND-GAr) is a high-pressure gaseous argon TPC operating at 0.5 T

- SAND (System for on-Axis Neutrino Detection) is a hybrid system with a baseline design that includes a three-dimensional projection scintillator tracker (3DST).

- Both ND-LAr and ND-GAr are movable from on-axis to off-axis (See next slide on PRISM).

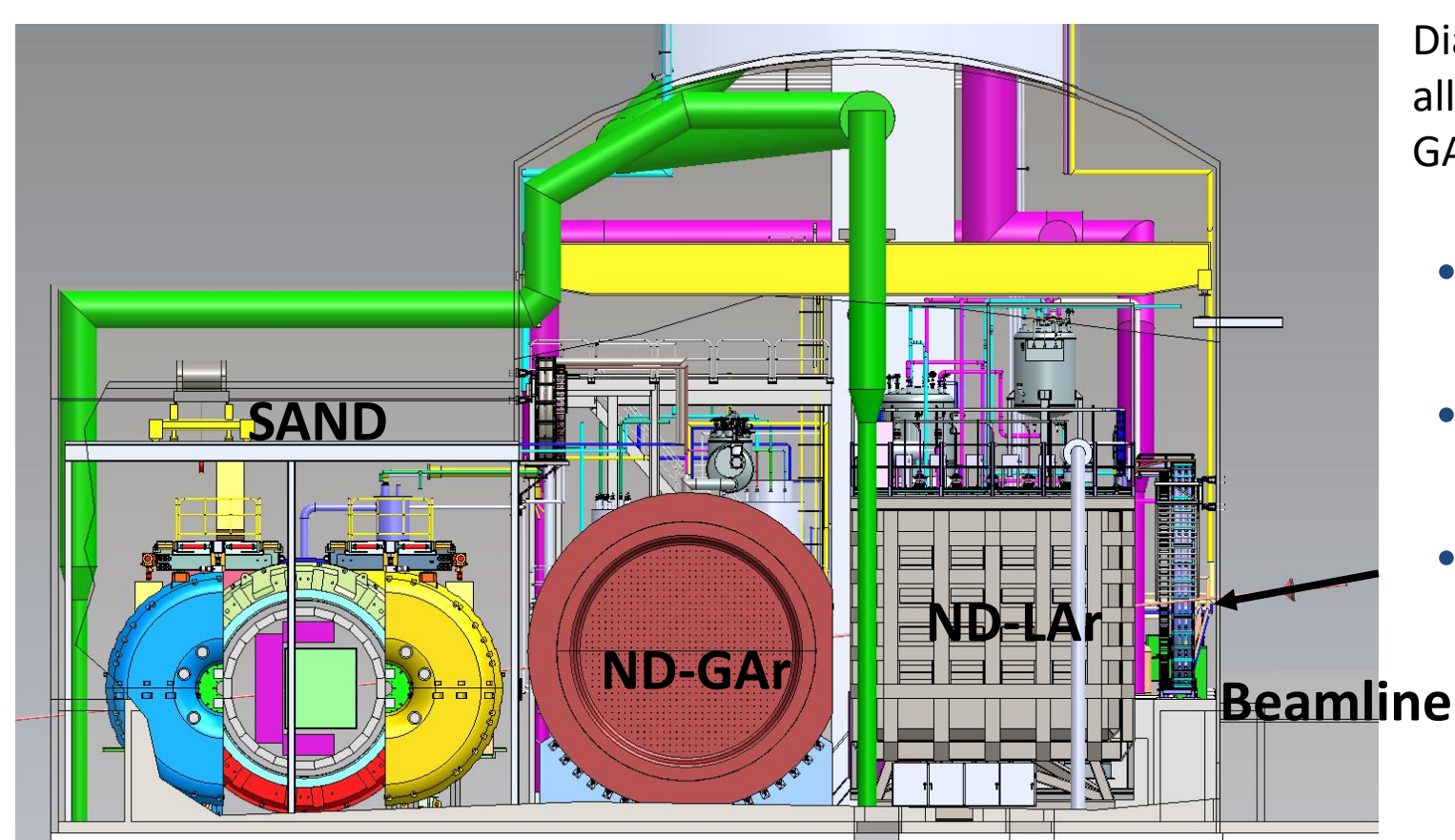

Diagram of DUNE Near Detector complex with

all three systems. In addition, ND-LAr and ND-

GAr can move off-axis.

- ND-LAr allows target for neutrino interactions with hadronic activity.

- ND-GAr allows for muon and sign reconstruction of events in ND-LAr

- SAND provides beamline monitoring with a detector and target that enables weekly beam-monitoring.

Rails to move off-axis. ND-LAr and ND"-GAr move into the page.

arxiv:2103.13910 


\section{DUNE PRISM}

- DUNE PRISM is a method to use the movement of the ND-LAr and ND-GAr detectors to better model cross sections and the spectrum of the DUNE neutrino beam.

- ND-LAr and ND-GAr can move $30 \mathrm{~m}$ off-axis (2.5 degrees).

- Allows probing of both flux and neutrino interaction modelling.

- Probing specific energy ranges and their exclusive channels.

- Enables flux-matching fits across the different off-axis locations.

- Linear combination of fluxes into a flux matrix.

- $\left|\mathrm{N}_{\text {flux }} \mathrm{C}-\mathrm{F}_{\text {flux }}\right|=0$ with coefficients (c), $\mathrm{N}$ is the ND flux, and $\mathrm{F}$ the FD flux.
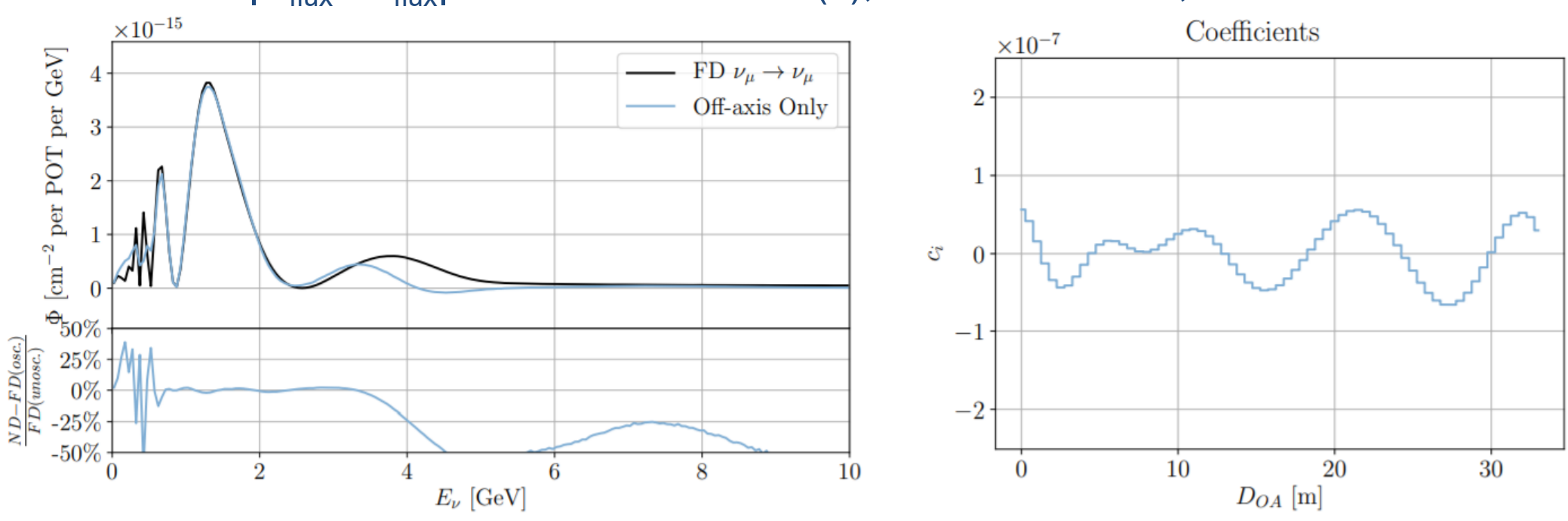

GENIE 2.12.10, DUNE FD TDR CV Tune

CC Inclusive - CC 1p1 $1 \mathrm{~h}+2 \mathrm{p} 2 \mathrm{~h}$

- CC Res $1 \pi \quad$ CCDIS

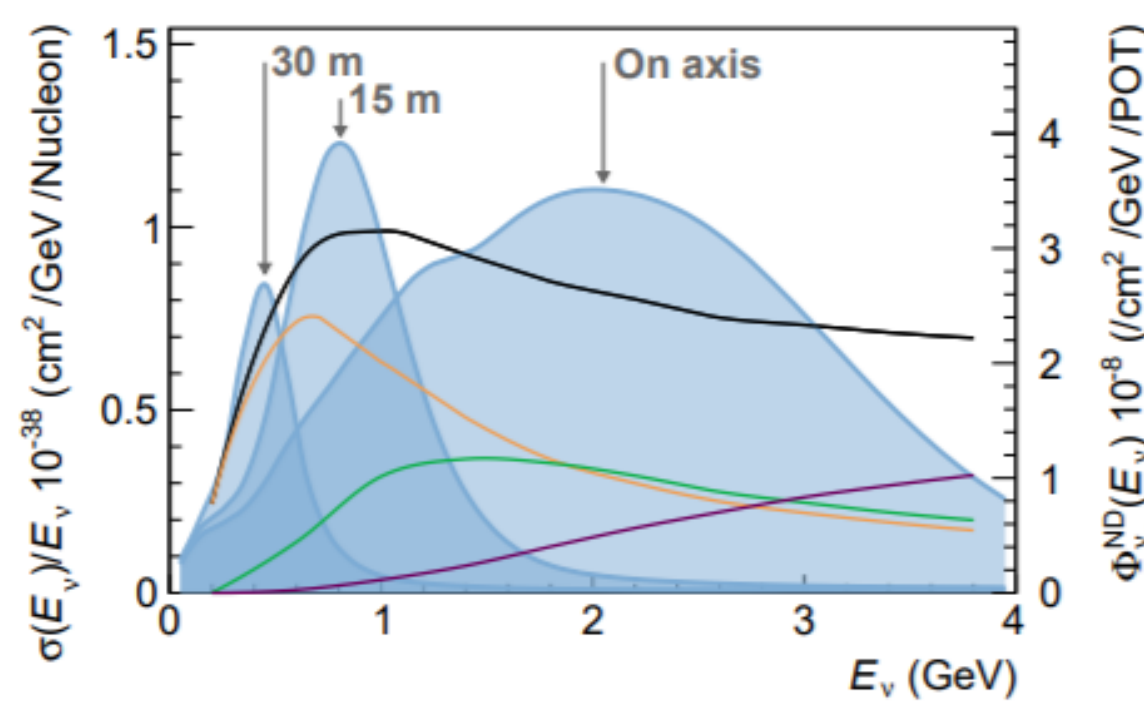

Spectrum of simulated neutrino events as a function of distance from on-axis at the DUNE Near Detector. 


\section{DUNE ND-LAr}

- Based on successful prototyping of a pixelized readout (JINST 13 P10007 2018).

- 35 modules of LAr TPCs making a 5-m long, 7-m wide, and 3-m high detector.

- Seven rows of five modules.

- Each module will contain 20 tiles of 10,240 readout pixels.

- Photon detection by use of ArCLight (Instruments 2, no. 1, 2018) and Light Collection Modules (LCMs).

- 60 ArCLight and 20 LCM per ND-LAr module.

- 35 segmented light readout systems allows pinpoint isolation of interaction.

- Average 10 us spill produces 55 neutrino interactions in and around the TPC.

- Light readout in a module can provide timing of approx. $25 \mathrm{~ns}$.

- Each cube will have a maximum drift time of approx. 300 microseconds (DUNE-FD roughly $2.3 \mathrm{~ms}$ ).

- Electric field technical spec. is $500 \mathrm{~V} / \mathrm{cm}$

o Demonstrated operationality at $1 \mathrm{kV} / \mathrm{cm}$.

\section{arxiv:2103.13910}

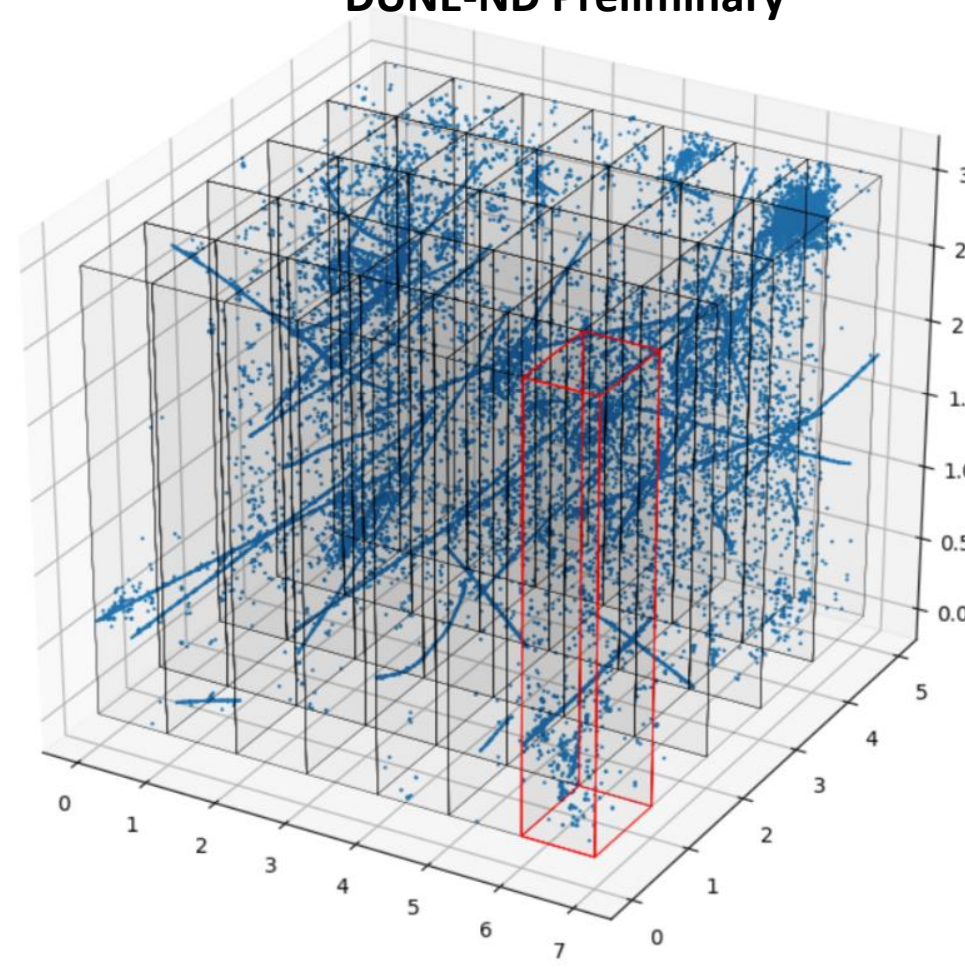

DUNE neutrino beam spill with pile-up. Modular segmentation allows for optical reconstruction and vertexing.

A mockup of the 50-cm long drift ND-LAr module. 


\section{Prototyping of DUNE ND-LAr}

Image of the demonstrator

- Current prototype is module 0 of the $2 \times 2$ demonstrator.

- Ran in spring of 2021.

- $2 \times 2$ demonstrator modules will be approximately $50 \%$ the design height and $70 \%$ design width.

- Neutrino data at Fermilab planned with the Fermilab NuMI beam in 2022.

\section{DUNE-ND Preliminary}

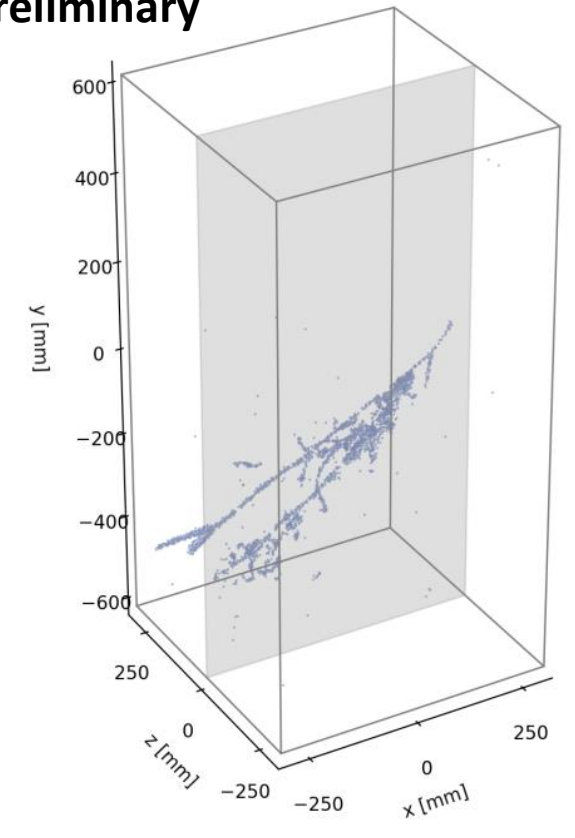

Cosmic ray event imaged in Module $02 \times 2$ demonstrator.

\section{DUNE-ND Preliminary}

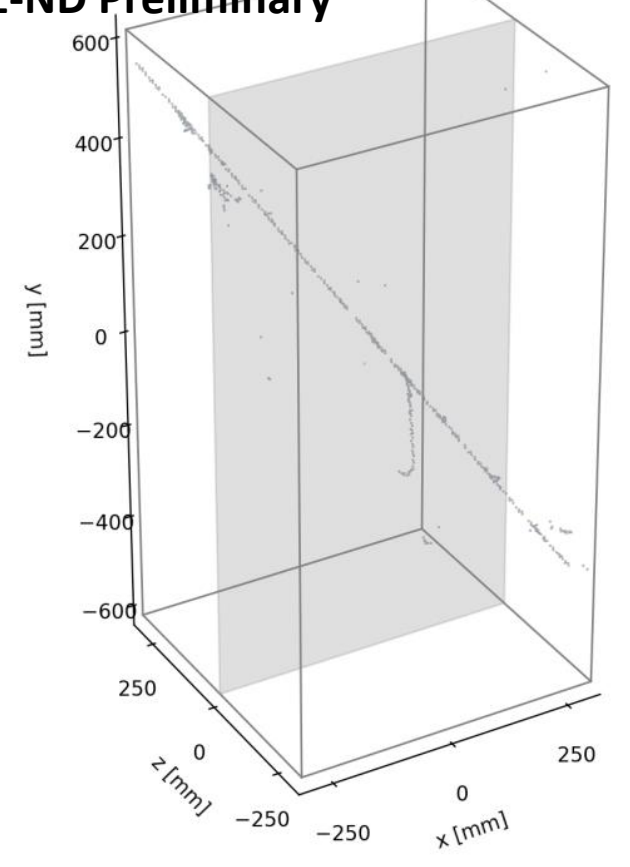

Picture of two anodes (ends) in field cage.

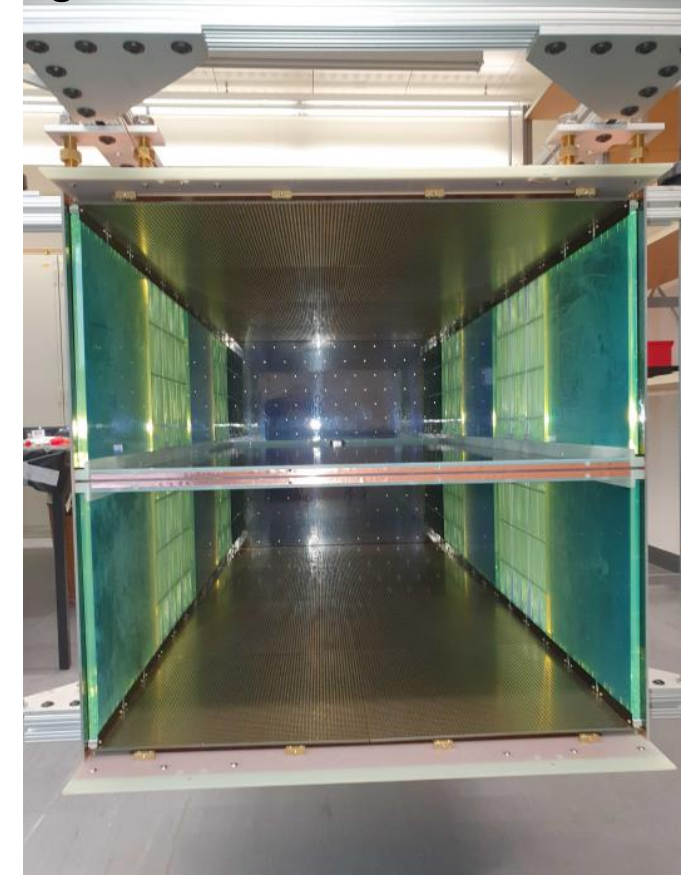




\section{Prototyping of DUNE ND-LAr}

Over 20 million events in approximately a week of data.

- Cosmic data with the demonstrator shows encouraging purity and reconstruction results.
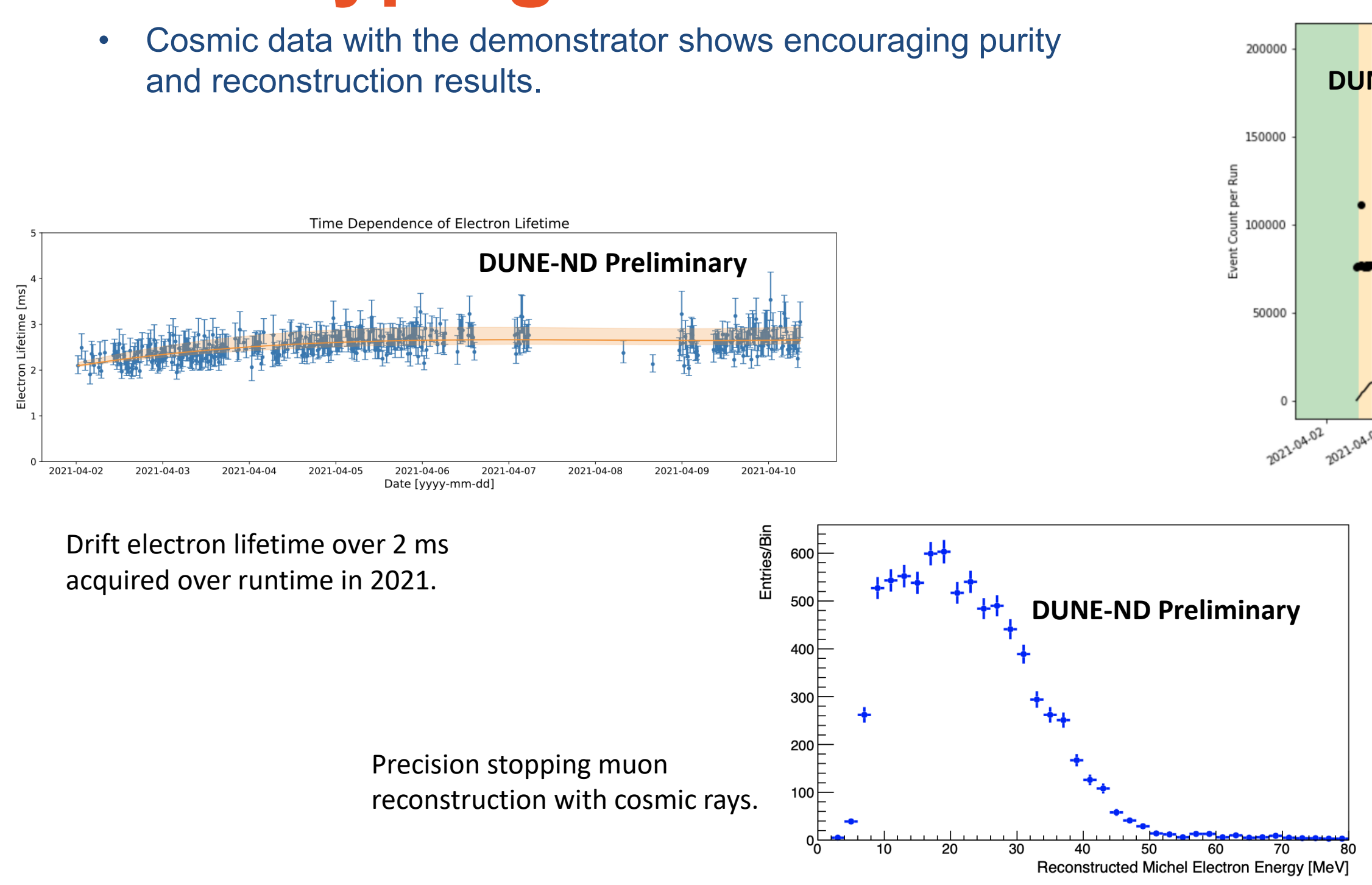

Drift electron lifetime over 2 ms acquired over runtime in 2021

Precision stopping muon reconstruction with cosmic rays.

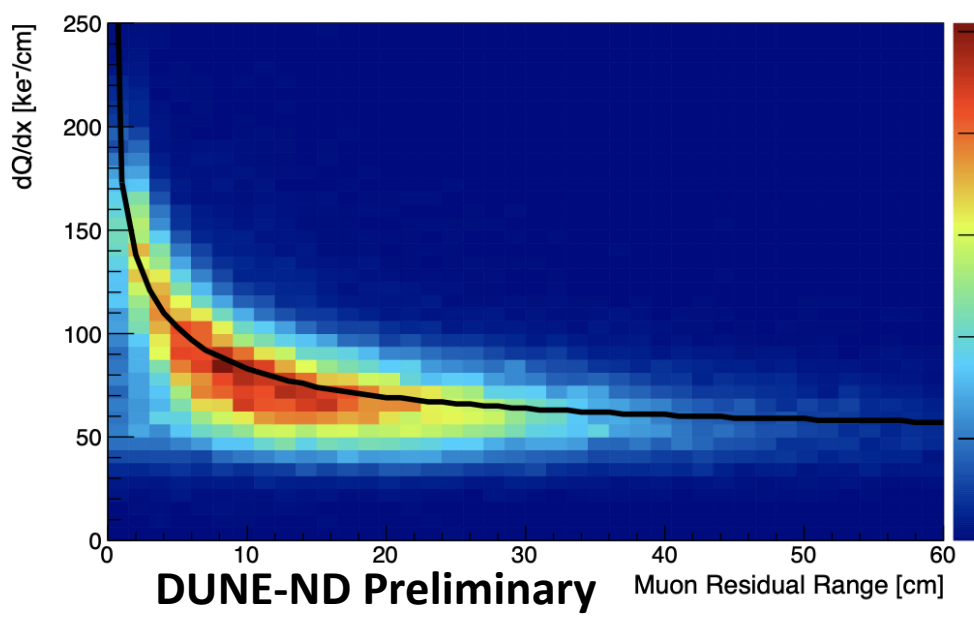




\section{DUNE ND-GAr}

- Based on ALICE TPC with a 5-m diameter that is 5.2-m long.

- Approx. 1-ton fiducial gas mass of argon ( $90 \%$ concentration) with $\mathrm{CH}_{4}$ mixture (10\% concentration).

- $97 \%$ of gas mixture mass is argon.

- Will be surrounded by an ECAL modelled after CALICE intended for photon reconstruction (JINST 5 (2010) P05004).

- Contains layers of lead and plastic scintillator.

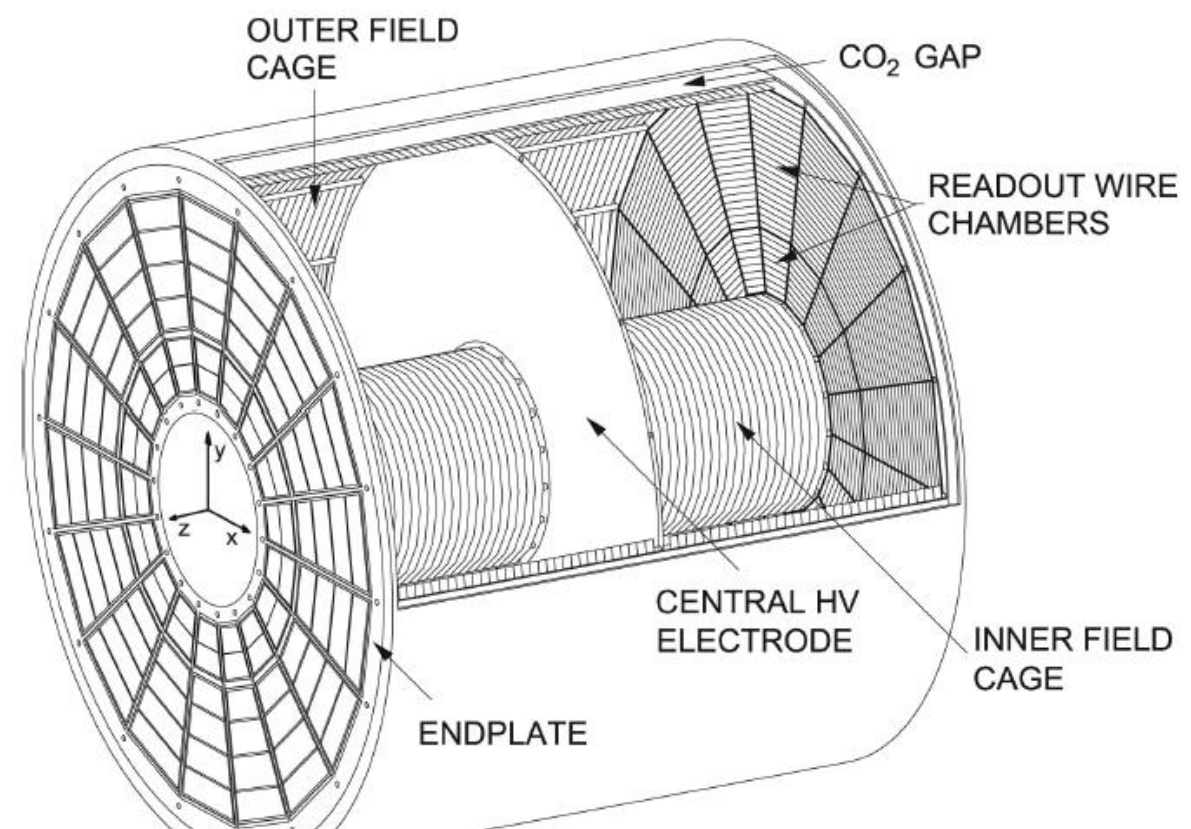

Diagram of ALICE TPC Nucl. Instrum. Meth. A622 (2010) 316-367.

Drawing of the full setup with the TPC in yellow and the ECAL in blue. A muon tagging system will also be included. 


\section{DUNE ND-GAr Reco Studies}

- Reconstruction available for ND-GAr simulation studies with evaluations of reconstruction efficiencies.

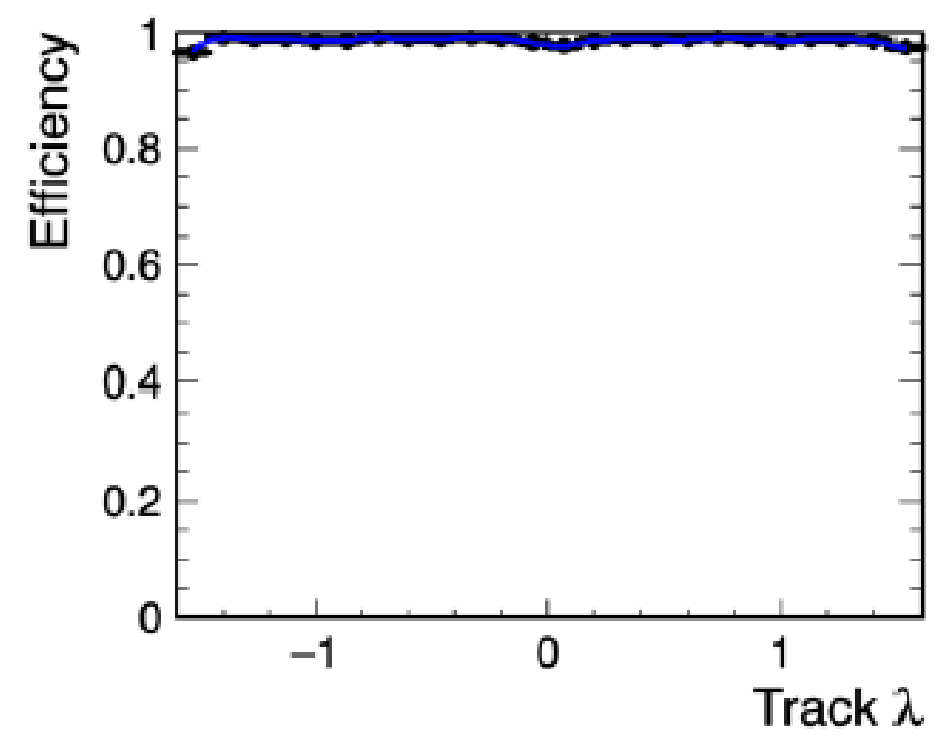

Efficiency in simulation of reconstructing tracks as a function of the angle relative to the central plane.

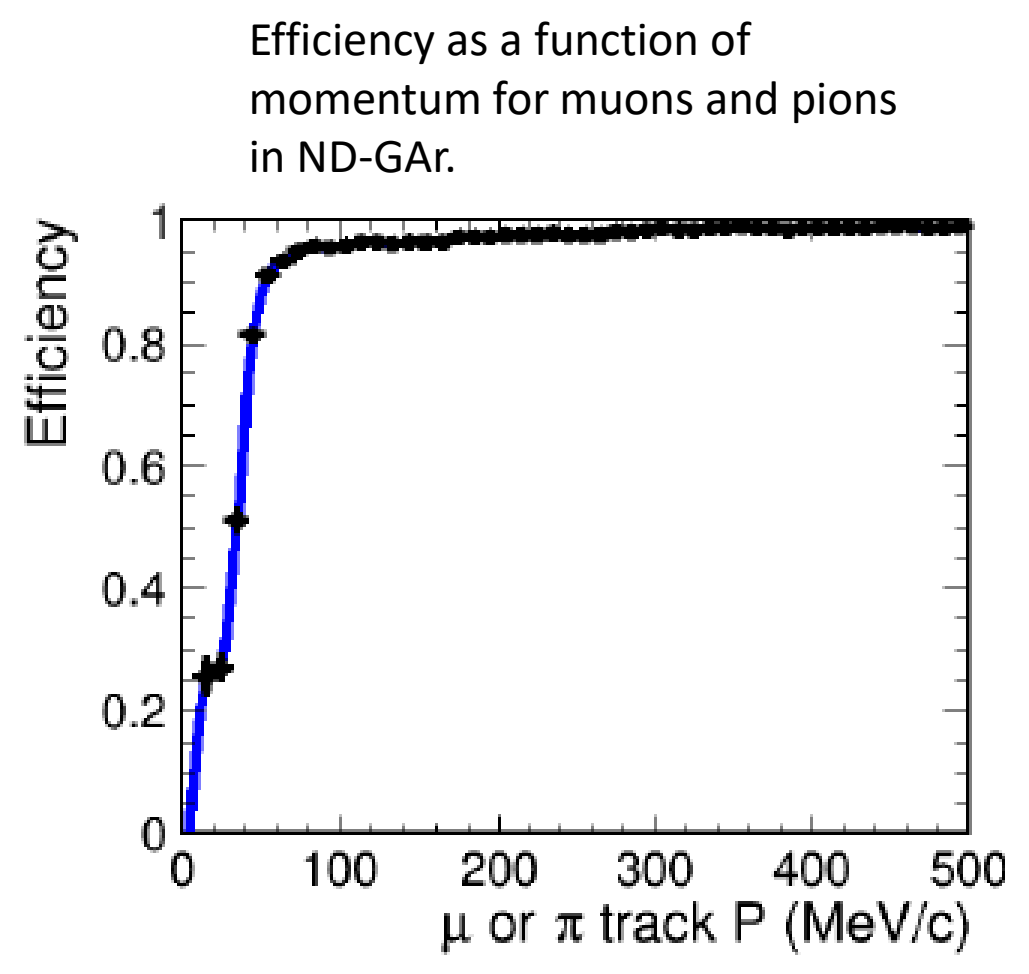

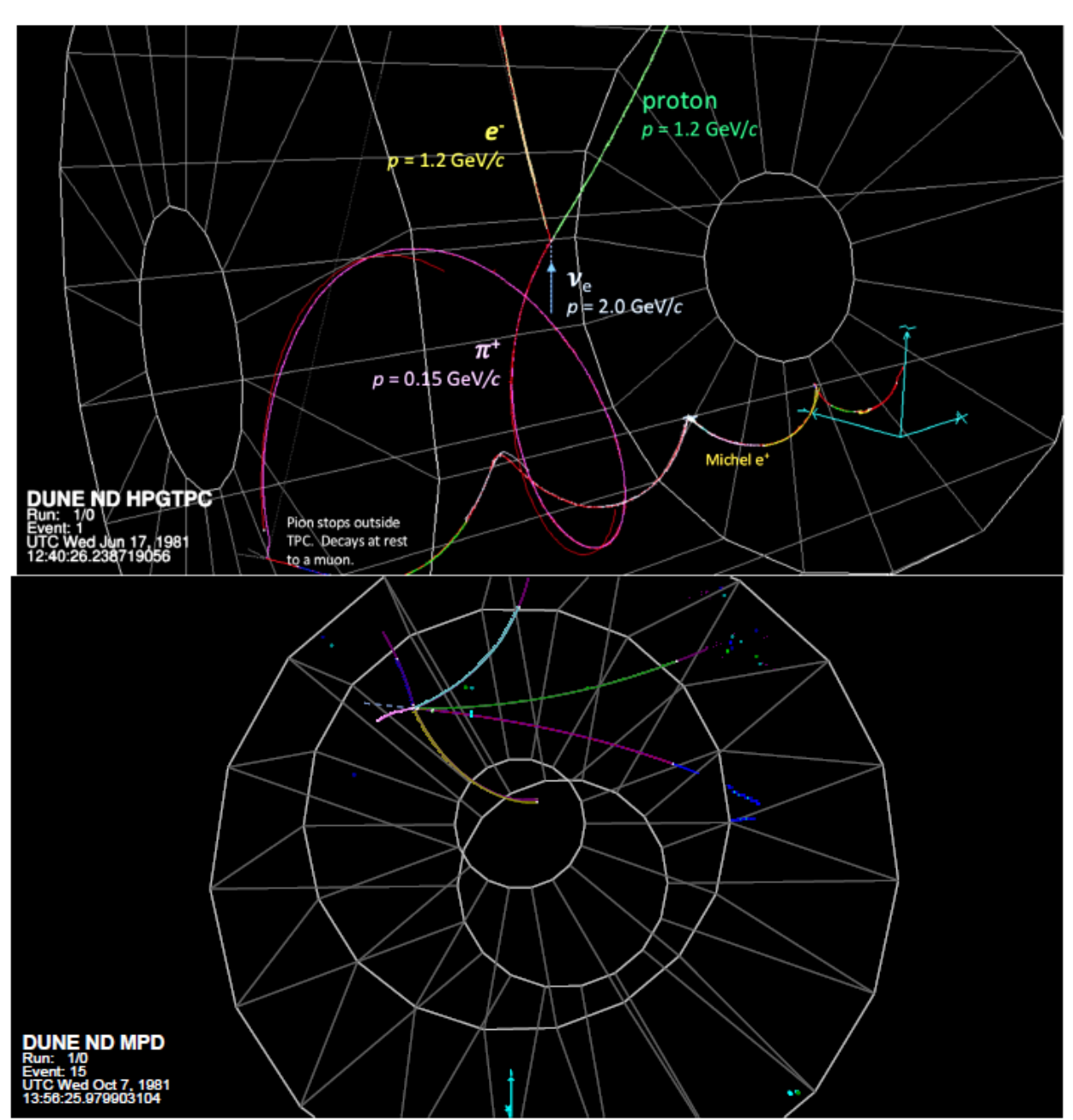

Simulated event display of an electric neutrino interaction with pion production (top) and of a neutrino interaction with

arxiv:2103.13910 five protons (bottom) 


\section{Near Detector SAND}

- Baseline design includes KLOE magnet (0.6 T).

- Small target of argon in front of the detector.

- Target large enough for weekly monitoring of DUNE beam performance (approx. 1 ton).

- Baseline component is 3DST.

- Will contain $(1.5 \mathrm{~cm})^{3}$ scintillating cubes with a mass of approx. 10 tons.

- Plans currently for 3DST to be surrounded by:

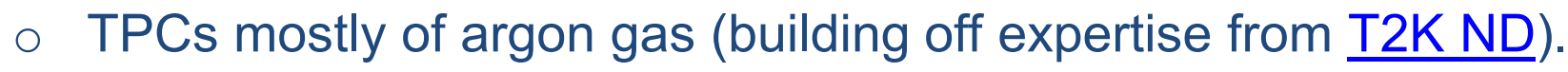

- Straw tracking tubes (STT).

- Alternative plans would have an STT-only SAND.

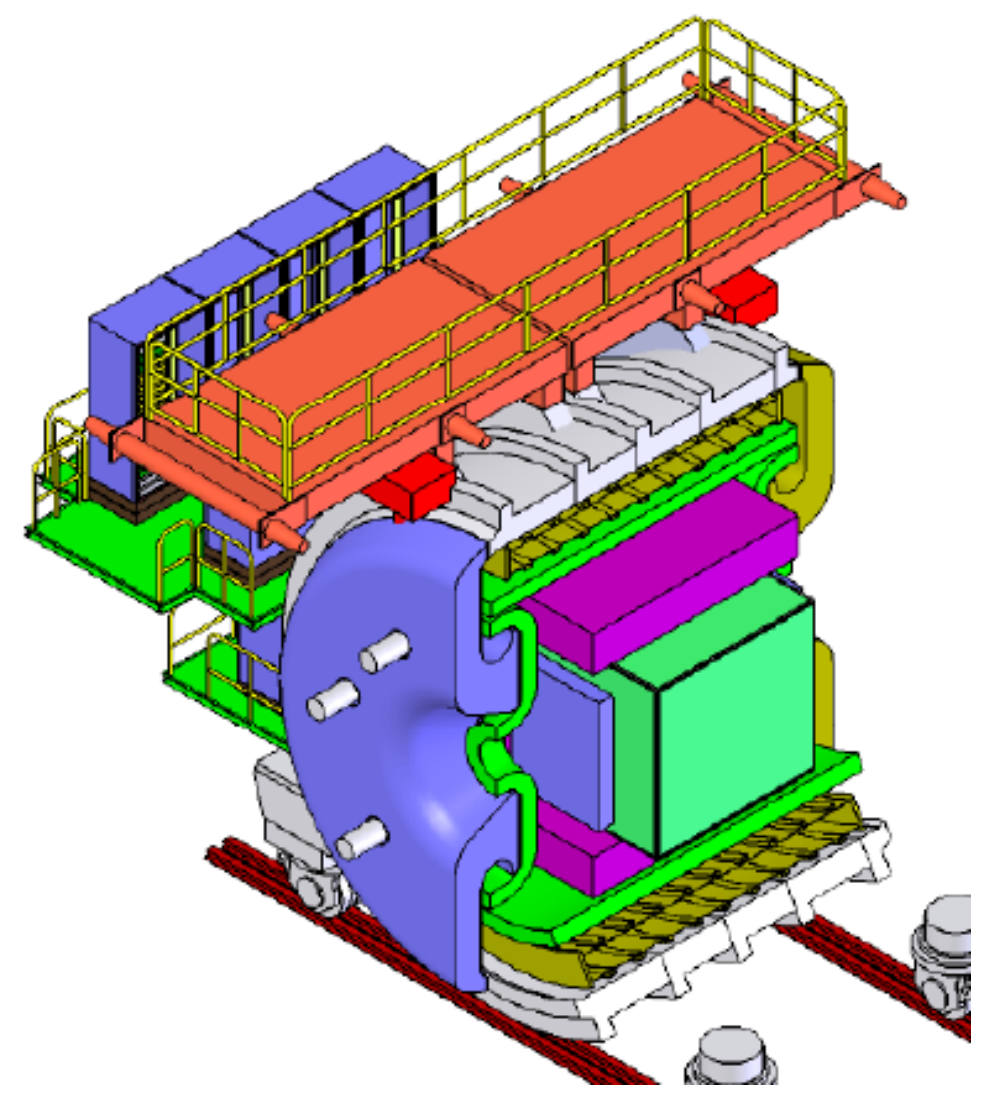

Diagram of the DUNE SAND Near Detector system with the KLOE magnet (blue), 3DST (green), and either the STT or TPC (purple). 


\section{Conclusion}

- DUNE uses three robust systems of detectors to understand the beam neutrino flux and cross section modelling of interactions on argon.

- ND-LAr and ND-GAr will move off-axis to allow for flux matching and precision cross section analyses across the beam spectrum.

- SAND provides beamline monitoring with the ability to probe the neutrino beam as a function of time.

All systems will provide the physics requirements necessary to provide the inputs for the DUNE long-baseline program. 


\section{Backup Slides}




\section{DUNE Near Detector Location}

- Main purpose to measure the un-oscillated neutrino beam that will be sent to the Far Detector site in South Dakota.

- Will also carryout neutrino cross section and BSM analyses.

- Spaced approx. $500 \mathrm{~m}$ from the target.
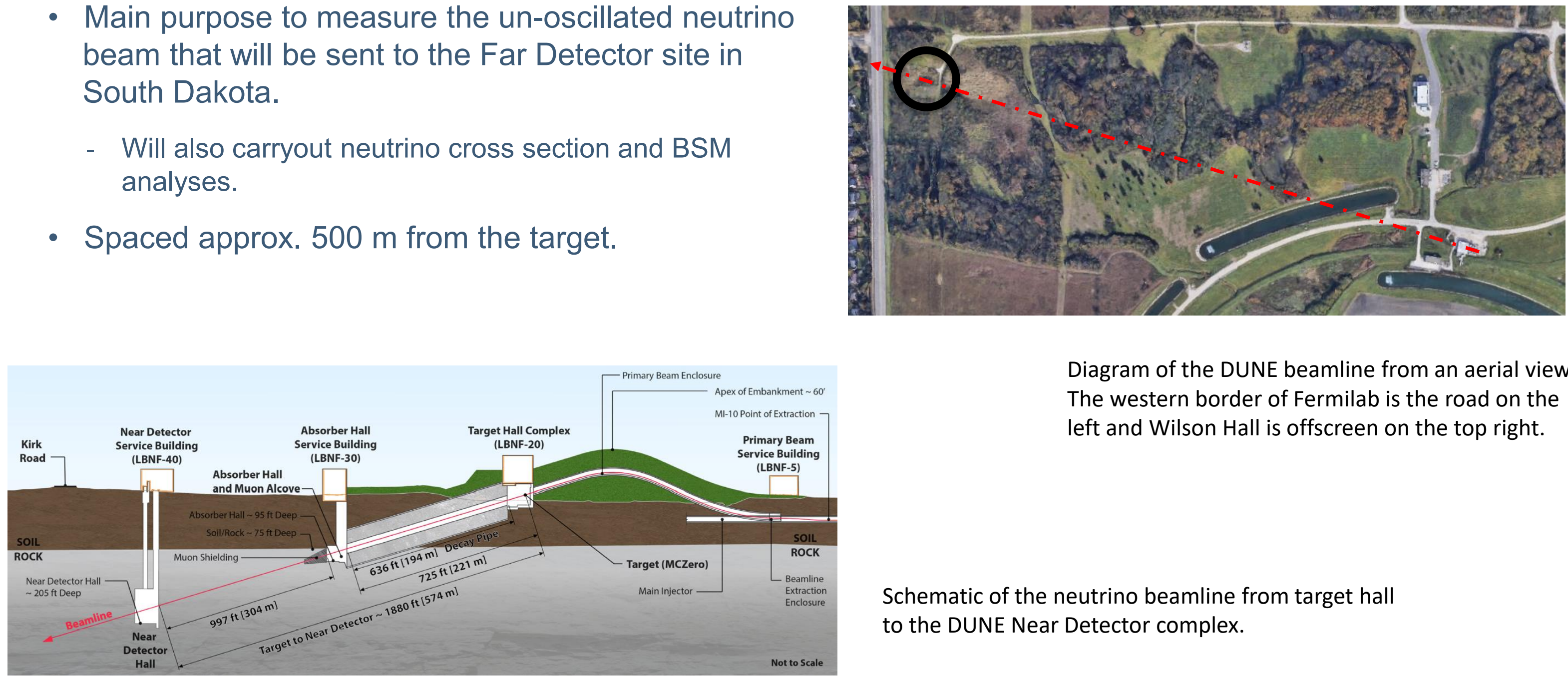

Diagram of the DUNE beamline from an aerial view. The western border of Fermilab is the road on the left and Wilson Hall is offscreen on the top right.

Schematic of the neutrino beamline from target hall to the DUNE Near Detector complex. 


\section{ND-LAr Light Detection}

- Both technologies are light traps using dielectrics with tetraphenyl butadiene to detect the deep UV scintillation light from argon.

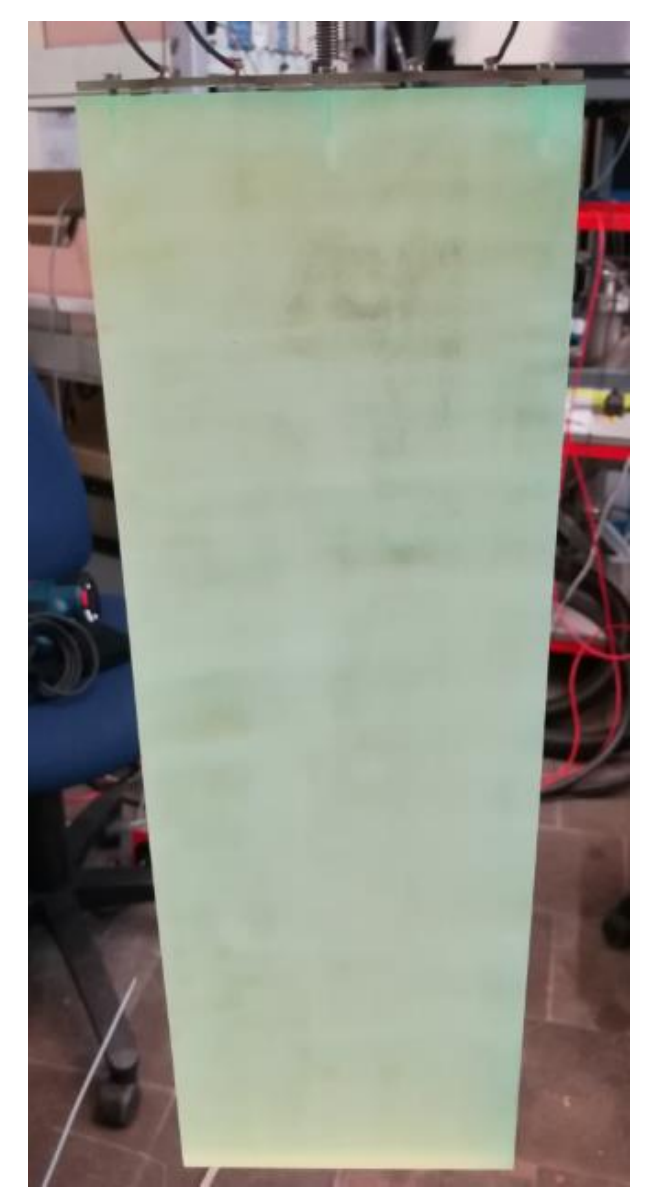

ArCLight (left) and LCM (right). The most obvious difference is the usage of a slab (left) and fibers (right).

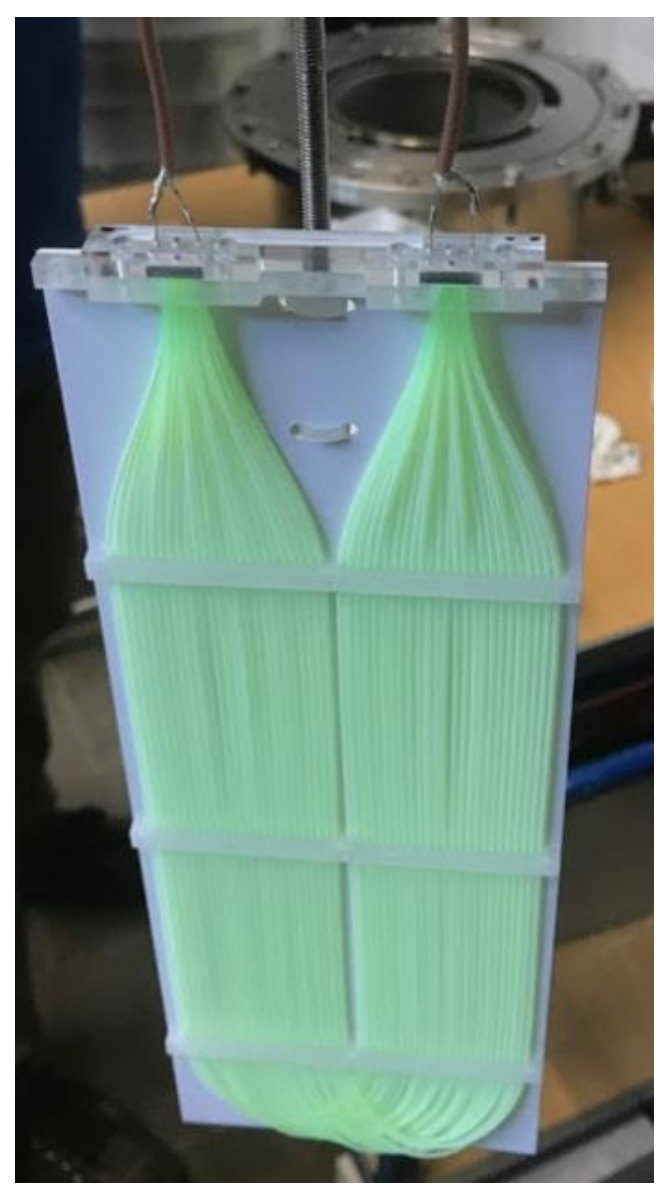

